Revista Iberoamericana de las Ciencias Biológicas y Agropecuarias

https://doi.org/10.23913/ciba.v8i16.90

Artículos Científicos

\title{
Factores socioeconómicos como impulsores de vulnerabilidad alimentaria
}

\section{Socioeconomic Factors as Drivers of Food Vulnerability}

Fatores socioeconômicos como motores da vulnerabilidade alimentar

Susana Marceleño Flores

Universidad Autónoma de Nayarit, México

smlmarcel@hotmail.com

https://orcid.org/0000-0003-0430-8128

Rebeca de Haro Mota

Universidad Autónoma de Nayarit, México

deharo@uan.edu.mx

https://orcid.org/0000-0001-6067-4013

Oyolsi Nájera González

Universidad Autónoma de Nayarit, México

oyolsi@uan.edu.mx

https://orcid.org/0000-002-8637-9482 


\section{Revista Iberoamericana de las Ciencias Biológicas y Agropecuarias}

\section{Resumen}

En este trabajo se evalúa la vulnerabilidad alimentaria en los municipios de Nayarit a partir de la construcción de un índice que considera tres variables: desarrollo socioeconómico, inseguridad alimentaria y vulnerabilidad alimentaria. Dentro de los resultados se encuentra que el municipio de Del Nayar se ubica en un nivel muy alto de vulnerabilidad; mientras que Huajicori, Acaponeta, Rosamorada y Ruiz en un nivel alto de vulnerabilidad. Por otra parte, los factores identificados como impulsores de vulnerabilidad son la deficiente dotación de carreteras pavimentadas, la baja actividad económica y el bajo nivel de escolaridad del jefe o jefa de familia.

Palabras clave: desarrollo socioeconómico, índice, inseguridad alimentaria, Nayarit, nivel de escolaridad.

\section{Abstract}

This work assesses the food vulnerability in the municipalities of Nayarit using an index of food vulnerability that incorporates three factors: socio-economic development, food insecurity and food vulnerability. Results show that the municipality of Del Nayar was found at very high vulnerability level; Huajicori, Acaponeta, Rosamorada and Ruiz at high vulnerability level. On the other hand, the factors identified as drivers of vulnerability are deficient road structure, low economic activity and low schooling of the household head.

Keywords: socio-economic development, index, food insecurity, Nayarit, schooling.

\section{Resumo}

Este trabalho avalia a vulnerabilidade alimentar nos municípios de Nayarit com base na construção de um índice que considera três variáveis: desenvolvimento socioeconômico, insegurança alimentar e vulnerabilidade alimentar. Entre os resultados, o município de Del Nayar está localizado em um nível muito alto de vulnerabilidade; enquanto Huajicori, Acaponeta, Rosamorada e Ruiz em um alto nível de vulnerabilidade. Por outro lado, os fatores identificados como vetores de vulnerabilidade são a baixa oferta de estradas pavimentadas, a baixa atividade econômica e o baixo nível de escolaridade do chefe de família. 


\section{Revista Iberoamericana de las Ciencias Biológicas y Agropecuarias}

Palavras-chave: desenvolvimento socioeconômico, índice, insegurança alimentar, Nayarit, nível de escolaridade.

Fecha recepción: Enero 2019

Fecha aceptación: Junio 2019

\section{Introducción}

Un índice es una agregación de un conjunto de indicadores individuales que da evidencia de un problema multifacético utilizando métodos matemáticos. Su principal objetivo es cuantificar y simplificar la información del conjunto de indicadores de manera que sea comprensible para todo tipo de público (Bas Cerdá, 2014). Con el tiempo, el número de índices aplicados a diferentes ámbitos de interés ha aumentado, ya que miden conceptos multidimensionales, son de importancia política y relevantes en la toma de decisiones (Granger, 2001); además, permiten establecer prioridades políticas (Saltelli, 2007).

La construcción de índices es gradual. Primero se encuentra al conjunto de indicadores simples que acumulan información existente; después, en un segundo nivel, están los indicadores temáticos: son indicadores simples agrupados según tema o área específica. Y en un tercer nivel se ubican los índices que se generan al combinar los indicadores temáticos, que se presentan como una medida compuesta única (Freudenberg, 2003). Al construir índices es necesario estandarizar las variables para ponerlas en una base común y evitar problemas con la mezcla de unidades de medida. Respecto a estandarizar, el enfoque de la desviación estándar tiene características deseables cuando se trata de agregación y es el más usado (Freudenberg, 2003), ya que todas las variables se convierten en una escala común y asume una distribución normal, con un promedio de cero, lo que significa que evita introducir alteraciones derivadas de las diferencias en las medias.

Los índices de vulnerabilidad en el tema alimentario han contribuido con el diseño de políticas de Estado para erradicar la desnutrición crónica infantil; permiten identificar y priorizar la atención a la población más vulnerable (Ministerio de Desarrollo e Inclusión Social [Midis] y Organización de las Naciones Unidas para la Alimentación y la Agricultura [FAO], 2012). 


\section{Revista Iberoamericana de las Ciencias Biológicas y Agropecuarias}

La definición de seguridad alimentaria, aceptada por el Comité de Seguridad Alimentaria Mundial (CSA), es la siguiente: "Acceso material y económico a alimentos suficientes para todos los miembros del hogar, sin correr riesgos indebidos de perder dicho acceso". Esta definición, según Thomson (1999), introduce el concepto de vulnerabilidad. Para el CSA, organismo perteneciente a la FAO, la vulnerabilidad de la seguridad alimentaria se refiere a toda la gama de factores que hacen que las personas queden expuestas a inseguridad alimentaria. Así, el análisis de vulnerabilidad alimentaria se ha desarrollado como una forma de identificar quién es incapaz de obtener alimentos y los factores que le restringen su acceso (Stephen y Downing, 2001).

En la evaluación de vulnerabilidad en el tema alimentario se han usado índices globales que se construyen con diversas variables; se han utilizado fuentes de información secundarias, como censos de población y vivienda, tasas de desnutrición crónica y pobreza extrema, todas ellas agrupadas por componentes de seguridad alimentaria (Midis y FAO, 2012). También para índices globales se han usado datos de pobreza, desarrollo humano e inseguridad alimentaria (Instituto Nacional de Estadística y Censos [INEC], 2004). En otro caso se usaron datos de producción agropecuaria, población, red vial, ingreso, pobreza, emigración, enfermedades, acceso a agua y analfabetismo, todos ellos referidos al componente de seguridad alimentaria (Dumazert, 2008).

Hasta la fecha muchos de los estudios sobre vulnerabilidad se han centrado en la pobreza (Chaudhuri y Datt, 2001; Holzmann y Jørgensen, 2000; Ozughalu y Ogwumike, 2013; Figueroa, 2005), lo que indica que la población de bajos recursos es la más vulnerable. Sin embargo, los modelos utilizados en estos análisis no incorporan la capacidad de adaptación y, por lo tanto, no consideran que la población desarrolla mecanismos para enfrentar eventos desfavorables. Por otra parte, centrándose únicamente en la pobreza, se excluyen las diversas estrategias que la población tiene para acceder a los alimentos. Asimismo, no identifica las preferencias alimenticias de la población.

En situación de crisis, la vulnerabilidad se relaciona con activos que pueden movilizar a los hogares o comunidades (Salomone, 2016). Es un concepto dinámico que cambia según diversos factores (Bengoa, 1996). De hecho, se ha considerado que el nivel de vulnerabilidad depende de la posesión de activos, también llamados capitales (Kaztman y Filgueira, 1999). Los capitales son aquellos recursos con los que cuenta una comunidad (Gutiérrez y Siles, 


\section{Revista Iberoamericana de las Ciencias Biológicas y Agropecuarias}

2008). Sin embargo, Filgueira y Peri (2004) y Kaztman (2000) consideran que además de los activos disponibles en los hogares se debe considerar cómo estos se relacionan con la estructura de oportunidades integradas por el Estado y la sociedad.

En su trabajo empírico, Moser (1998) encuentra que cuantos más activos tenga una persona o comunidad es menos vulnerable. En este sentido, el concepto de activos en este trabajo se asocia con capacidad adaptativa.

Una manera completa de abordar la vulnerabilidad es la propuesta por Hughes et al. (2012). En esta se incluyen a la exposición y la sensibilidad de un sistema a estresores simples o múltiples y la capacidad del sistema a ajustarse o capitalizarse exitosamente a los efectos de estos estresores.

En el marco de las consideraciones anteriores, al analizar integralmente la vulnerabilidad alimentaria es importante incorporar componentes relevantes que contribuyen a la vulnerabilidad, incluyendo la exposición, la sensibilidad y la capacidad adaptativa; así como factores de desarrollo socioeconómico y de seguridad alimentaria estimada a partir de la experiencia en hogares. Asimismo, al integrar todas estas dimensiones — vulnerabilidad, desarrollo socioeconómico y seguridad alimentaria-, es importante vincularlas con los elementos de seguridad alimentaria (disponibilidad, acceso, estabilidad y utilización).

El objetivo principal de este trabajo fue identificar factores impulsores de vulnerabilidad alimentaria en los municipios del estado de Nayarit, partiendo de un índice de impulsores socioeconómicos de la vulnerabilidad a la inseguridad alimentaria que considera variables socioeconómicas, de seguridad alimentaria y vulnerabilidad alimentaria.

En Nayarit se identifican tres municipios en condiciones socioeconómicas desfavorables: Del Nayar, La Yesca y Huajicori; ahí se concentra la mayor parte de la población indígena del estado. Estos tres municipios, que se localizan en la zona serrana y se distribuyen en $42 \%$ de la superficie estatal, están conformados por 1037 localidades dispersas y de difícil acceso, distantes de los centros de actividad económica y de comercialización. El Consejo Nacional de Evaluación de la Política de Desarrollo Social [Coneval] (2017) reporta esta zona con los rangos más altos (75 \%-100 \%) de población en situación de pobreza. Del Nayar, el municipio con mayor pobreza, ocupó el lugar 41 para el año 2015, con 23916 personas en pobreza extrema. 


\section{Revista Iberoamericana de las Ciencias Biológicas y Agropecuarias}

\section{Material y método}

Primeramente, se elaboró un modelo causal de la vulnerabilidad alimentaria. Se determinó que los componentes que reflejan la realidad de la vulnerabilidad están representados por los índices temáticos: desarrollo socioeconómico, inseguridad alimentaria y vulnerabilidad alimentaria. Finalmente, integrando estos índices temáticos, se construyó el "Índice de impulsores socioeconómicos de la vulnerabilidad a la inseguridad alimentaria" (ISEV), como se muestra en el esquema metodológico en la figura 1.

Figura 1. Proceso metodológico del ISEV

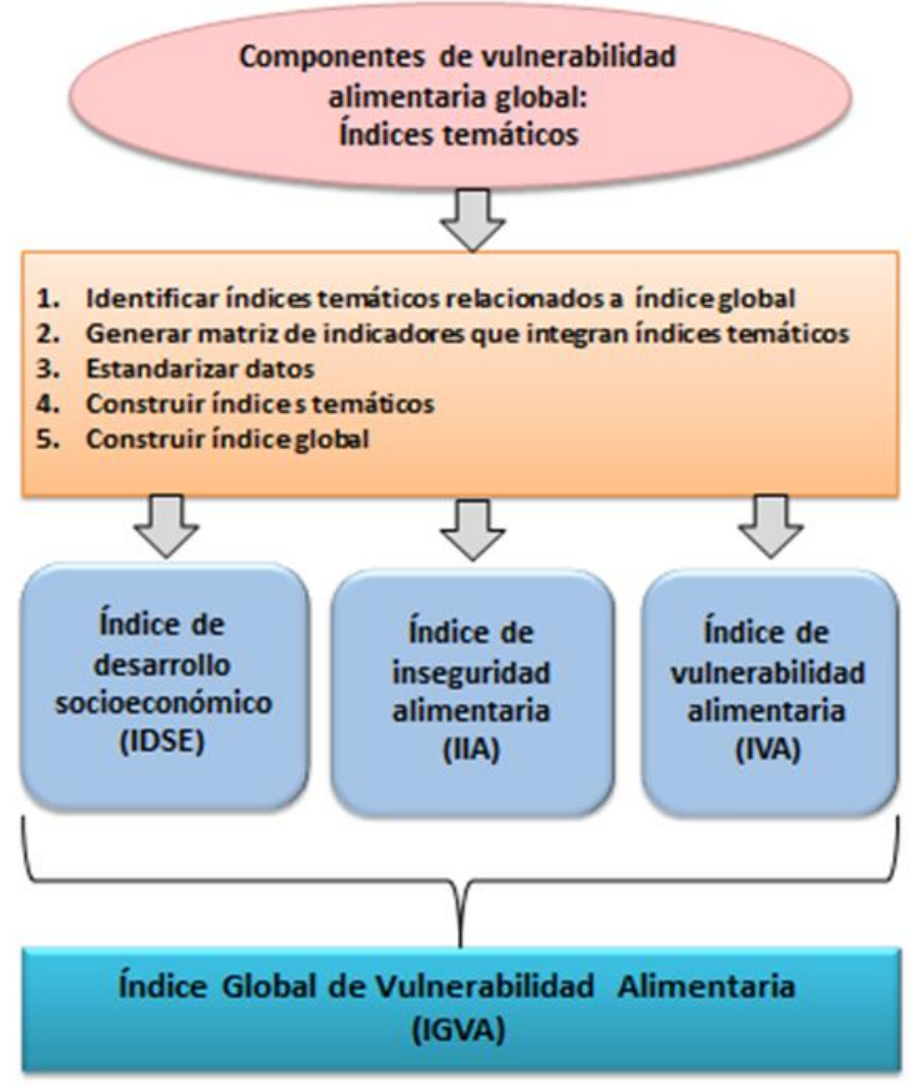

Fuente: Elaboración propia

Así, la evaluación de la vulnerabilidad alimentaria en los municipios de Nayarit se hizo a partir del ISEV, que se obtuvo promediando los índices de desarrollo socioeconómico, inseguridad alimentaria y vulnerabilidad alimentaria. El valor del índice de desarrollo socioeconómico se multiplicó por -1 antes de promediarlo, ya que tiene un significado inverso a la vulnerabilidad alimentaria. La relación funcional fue: 


\section{Revista Iberoamericana de las Ciencias Biológicas y Agropecuarias}

$$
I S E V=f\left(I D S E^{-}, I I A^{+}, I V A^{+}\right)
$$

Donde:

$I S E V=$ "Índice de impulsores socioeconómicos de la vulnerabilidad a la inseguridad alimentaria";

IDSE = índice de desarrollo socioeconómico;

$I I A=$ índice de inseguridad alimentaria;

$I V A=$ índice de vulnerabilidad alimentaria.

Se realizó una prueba de Bartlett para determinar si hay relación entre las variables que integran cada uno de los índices temáticos, así como el test de Kaiser-Meyer-Olkin (KMO) para conocer su grado de relación. Y se obtuvieron valores aceptables superiores a 0.50 con $p$ $<0.0001$.

Finalmente, se clasificaron los municipios en cinco niveles de acuerdo con el valor de cada índice: muy bajo (menor a -0.75 ), bajo (de $\geq-0.75$ a -0.25 ), medio (de $>-0.25$ a 0.25 ), alto (de $>0.25$ a 0.75 ) y muy alto (mayor a 0.75 ).

A continuación, se describen los materiales y el método para cada uno de los índices temáticos que integran el ISEV.

\section{Índice de desarrollo socioeconómico (IDSE)}

Este índice se formó con los siguientes indicadores: índice de marginación, grado de urbanización, tasa bruta de actividad económica, coeficiente de dependencia económica y densidad de carreteras pavimentadas. Para construir estos indicadores se usaron nueve variables que fueron tomadas del Instituto Nacional de Estadística y Geografía (Inegi) y del Consejo Nacional de Población (Conapo); todas ellas del año 2010. La relación funcional fue la siguiente:

$$
I D S E=f\left(M^{-}, G U^{+}, T B A E^{+}, C D E^{-}, D C^{+}\right)
$$

Donde:

IDSE = índice de desarrollo socioeconómico;

$M=$ índice de marginación;

$G U=$ grado de urbanización;

$T B A E$ = tasa bruta de actividad económica; 


\section{Revista Iberoamericana de las Ciencias Biológicas y Agropecuarias}

$C D E=$ coeficiente de dependencia económica;

$D C=$ densidad de carreteras pavimentadas.

A continuación, se calculó el índice de cada municipio promediando los cinco indicadores. El valor del índice de marginación y del coeficiente de dependencia económica se multiplicaron por -1 antes de promediarlos, porque tienen un significado inverso al desarrollo. Finalmente, de acuerdo con el valor del índice, se clasificaron los municipios en cinco niveles de desarrollo socioeconómico: muy bajo (menor a -0.50 ), bajo (de -0.50 a 0.00 ), medio (de 0.01 a 0.50 ), alto (de 0.51 a 1.00 ) y muy alto (mayor a 1.00 ).

\section{Índice de inseguridad alimentaria (IIA)}

El IIA se construyó a partir de los resultados de las encuestas a hogares que se aplicaron a amas de casa; el instrumento aplicado fue la Escala Latinoamericana y Caribeña de Seguridad Alimentaria (ELCSA). A partir de los resultados de la encuesta, primero se estimó un índice de inseguridad alimentaria en cada municipio, que representa la proporción de hogares con inseguridad alimentaria. Después, estos índices se transformaron en valores normalizados de una distribución caracterizada por los argumentos media y desviación

estándar. La fórmula empleada para estandarizar fue: $Z=\frac{X-\mu}{\sigma}$; donde $X$ es el valor que se desea normalizar, $\mu$ la media aritmética y $\sigma$ la desviación estándar. Finalmente, se clasificaron los municipios en cinco niveles, de acuerdo con el valor de cada índice: muy bajo (menor a 0.75 ), bajo (de $\geq-0.75$ a -0.25 ), medio (de $>-0.25$ a 0.25 ), alto (de $>0.25$ a 0.75 ) y muy alto (mayor a 0.75 ).

\section{Índice de vulnerabilidad alimentaria (IVA)}

El IVA se formó con los índices puestos a continuación: exposición, sensibilidad y capacidad adaptativa. El índice de capacidad adaptativa se construyó a partir de indicadores de capital humano, financiero, físico, social y natural.

La exposición la constituye la población más expuesta a sufrir inseguridad alimentaria. Para el índice de exposición se usaron cuatro indicadores construidos a partir variables tomadas del Inegi y del Conapo, todas ellas, una vez más, del año 2010. El índice de sensibilidad, que caracteriza la condición que hace que las personas sean susceptibles a sufrir inseguridad alimentaria, se construyó con cinco indicadores que se formaron a partir de 


\section{Revista Iberoamericana de las Ciencias Biológicas y Agropecuarias}

información de la Secretaría de Salud de Nayarit (SSN) del año 2011 y del Coneval en el año 2010. Para el índice de capacidad adaptativa, el cual representan el potencial, habilidades y recursos para hacer frente a la inseguridad alimentaria, se utilizó información de la Secretaría de Desarrollo Social (Sedesol) del año 2013 y del Inegi de los años 2010, 2012 y 2013.

Antes de integrar los índices exposición, sensibilidad y capacidad adaptativa, todos los indicadores empleados se transformaron en valores estandarizados de una distribución caracterizada por los argumentos media y desviación estándar. La fórmula empleada fue: $Z=$

$\frac{X-\mu}{\sigma}$; donde $X$ es el valor que se desea normalizar, $\mu$ la media aritmética y $\sigma$ la desviación estándar.

Finalmente, para obtener el IVA se sumaron el índice de exposición y el de sensibilidad, a esta suma se le restó el índice de capacidad adaptativa. La relación funcional del IVA fue como sigue:

$$
I V A=\text { exposición }+ \text { sensibilidad }- \text { capacidad adaptativa }
$$

\section{Resultados y discusión}

En los seis municipios identificados con alta o muy alta vulnerabilidad habitan 157832 personas que representan $15 \%$ de la población estatal; en los cinco municipios con vulnerabilidad media habitan 246859 personas (23\% de la población); por último, 680288 habitantes $(62 \%)$ distribuidos en nueve municipios presentan baja o muy baja vulnerabilidad alimentaria. Por su parte, los factores generales identificados como impulsores de vulnerabilidad fueron: deficiente dotación de carreteras pavimentadas, baja actividad económica y bajo nivel de escolaridad del jefe o jefa de familia.

Los resultados de las estimaciones expresadas en índices se presentan en la tabla 1, donde resalta que el valor más alto del ISEV (2.10) es considerablemente superior al que le sigue (0.73). Por lo demás, los valores de este índice se distribuyen de manera regular. 
Revista Iberoamericana de las Ciencias Biológicas y Agropecuarias

Tabla 1. ISEV por variables y municipios

\begin{tabular}{|l|c|c|c|c|}
\hline & IDSE & VA & IA & IGVA \\
\hline Del Nayar & -2.75 & 1.01 & 2.54 & 2.10 \\
\hline Jala & -0.01 & 0.49 & 1.70 & 0.73 \\
\hline Acaponeta & 0.00 & 0.95 & 0.54 & 0.50 \\
\hline Rosamorada & -0.45 & 0.41 & 0.55 & 0.47 \\
\hline Ruíz & 0.17 & 0.92 & 0.43 & 0.39 \\
\hline Huajicori & -1.01 & 0.34 & -0.34 & 0.33 \\
\hline Tecuala & 0.12 & 0.52 & 0.27 & 0.22 \\
\hline Tuxpan & 0.62 & -0.46 & 1.12 & 0.01 \\
\hline Compostela & 0.36 & 0.05 & -0.22 & -0.18 \\
\hline Santiago Ixcuintla & 0.23 & -0.03 & -0.40 & -0.22 \\
\hline La Yesca & -1.30 & -0.54 & -1.48 & -0.24 \\
\hline Santa María del Oro & 0.07 & -0.35 & -0.43 & -0.28 \\
\hline Ahuacatlán & 0.17 & -0.66 & -0.04 & -0.29 \\
\hline San Blas & 0.76 & -0.27 & 0.16 & -0.29 \\
\hline Ixtlán del Río & 0.48 & 0.59 & -1.51 & -0.47 \\
\hline Bahía de Banderas & 0.97 & 0.01 & -0.47 & -0.48 \\
\hline San Pedro Lagunillas & 0.14 & -1.15 & -0.16 & -0.48 \\
\hline Tepic & 1.08 & -0.27 & -0.20 & -0.52 \\
\hline Amatlán de Cañas & 0.08 & -1.25 & -1.33 & -0.89 \\
\hline Xalisco & 0.44 & -0.25 & -0.71 & -0.47 \\
\hline
\end{tabular}

Fuente: Elaboración propia

La jerarquización de los niveles de vulnerabilidad muestra la situación de cada uno de los municipios. En la figura 2 se observa que Del Nayar es el único municipio con muy alta vulnerabilidad alimentaria global. Los cinco municipios con alta vulnerabilidad son Huajicori, Acaponeta, Rosamorada, Ruíz y Jala; en tanto que Tecuala, Tuxpán, Santiago Ixcuintla, Compostela y La Yesca clasifican con vulnerabilidad media; enseguida están San Blas, Tepic, Xalisco, Santa María del Oro, San Pedro Lagunillas, Ixtlán del Río, Ahuacatlán y Bahía de Banderas con baja vulnerabilidad alimentaria, y, por último, está Amatlán de Cañas con muy baja vulnerabilidad alimentaria global. Asimismo, los resultados muestran que la condición de vulnerabilidad a nivel municipal tiene diversas causas. 
Figura 2. ISEV de Nayarit, 2015

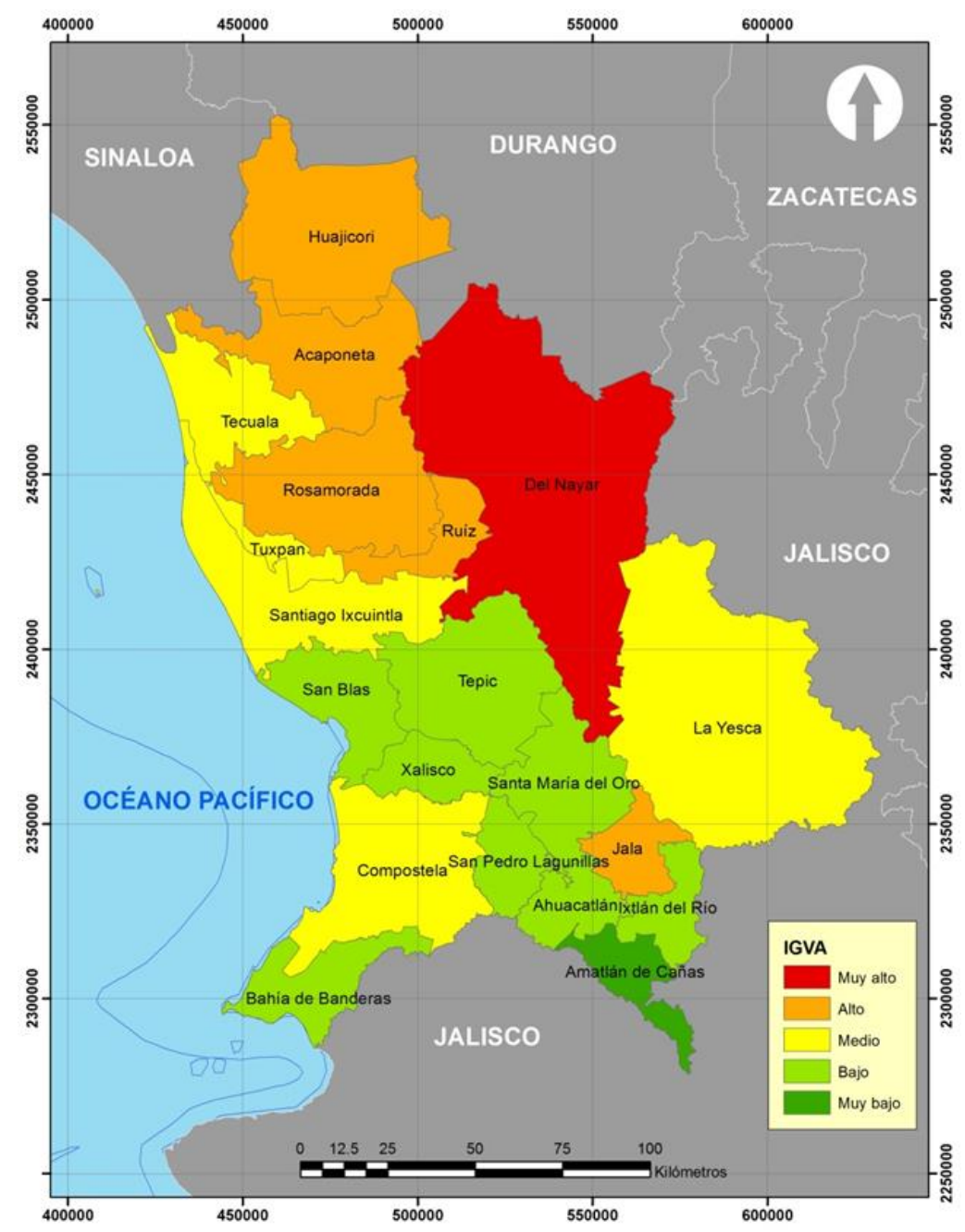

Fuente: Elaboración propia

\section{Condición de vulnerabilidad por municipio}

Del Nayar es el único municipio identificado con un ISEV muy alto. Tiene las condiciones con menor ventaja, índices de inseguridad y vulnerabilidad alimentaria muy altos y un IDSE muy bajo. Se caracteriza por ser de condición rural en la totalidad de sus localidades, con muy altos índices de marginación (3.6); presenta tasa bruta de actividad económica muy baja, solo $16 \%$ de la población está económicamente activa ocupada, cada 502 personas inactivas (sin empleo formal) son sostenidas por cada 100 activos (con empleo formal). Además, en Del Nayar se registró el mayor porcentaje de hogares con inseguridad alimentaria en el estado (45\%). También se identifica un nivel de vulnerabilidad alimentaria 


\section{Revista Iberoamericana de las Ciencias Biológicas y Agropecuarias}

muy alto (ver figura 3), $51 \%$ de la población tiene menos de 18 años, el promedio de escolaridad es el menor en el estado (4.5 años).

Figura 3. Índice temático de vulnerabilidad alimentaria de Nayarit, 2015

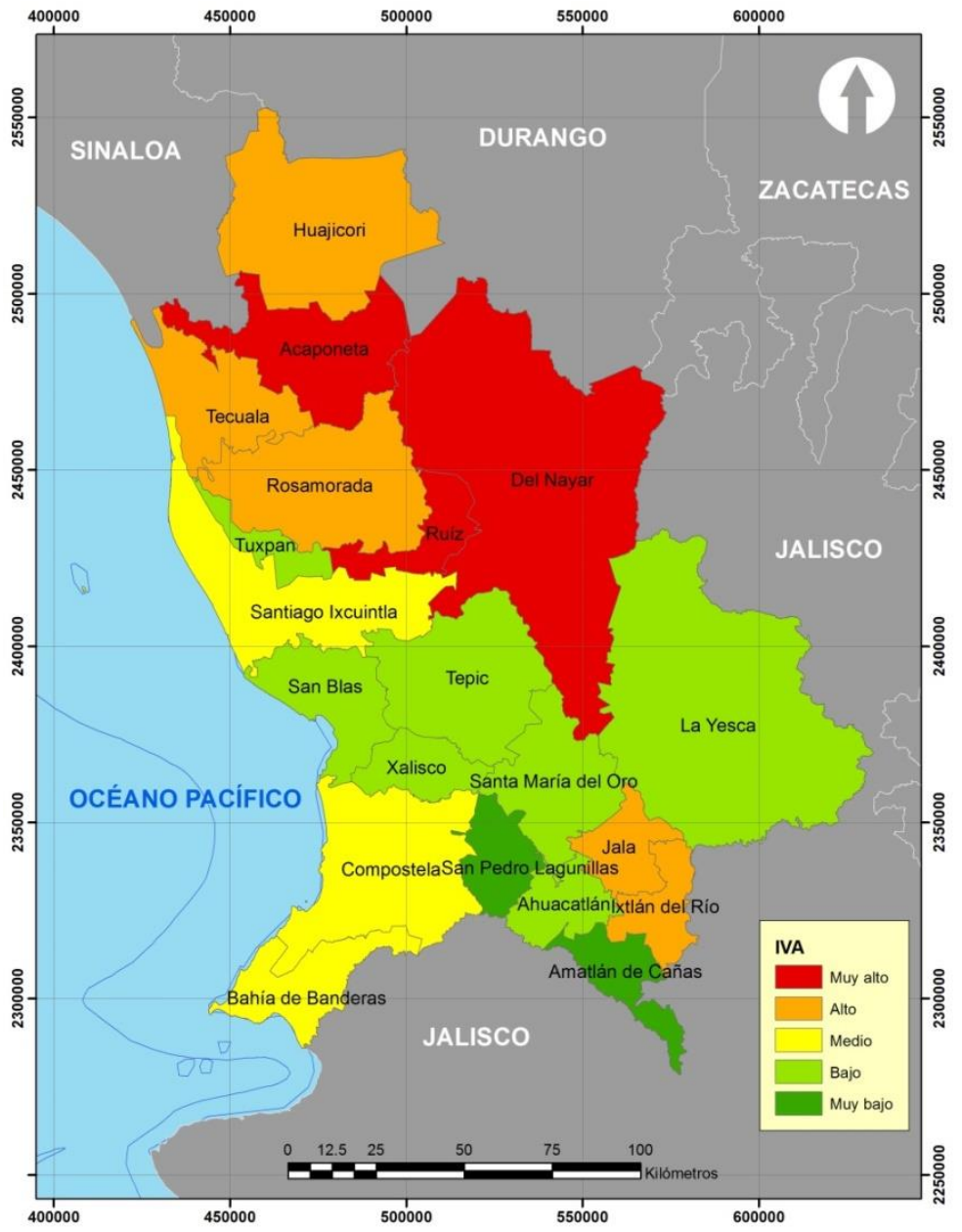

Fuente: Elaboración propia

Jala se identificó con un ISEV alto. Este municipio tiene índices de desarrollo socioeconómico bajo, inseguridad alimentaria muy alto y de vulnerabilidad alimentaria alto. La situación de desarrollo se debe a que tiene baja actividad económica, ya que solo $34 \%$ de la población se encuentra económicamente activa y además presenta alta dependencia económica (19 personas dependen de 10). Por otra parte, Jala, con $38.5 \%$ de la población con inseguridad alimentaria moderada o severa, se coloca en el segundo lugar de la entidad con inseguridad alimentaria (véase figura 4). Lo que coloca a este municipio con un IVA alto es que presenta alta sensibilidad. 
Figura 4. Índice temático de inseguridad alimentaria de Nayarit, 2015

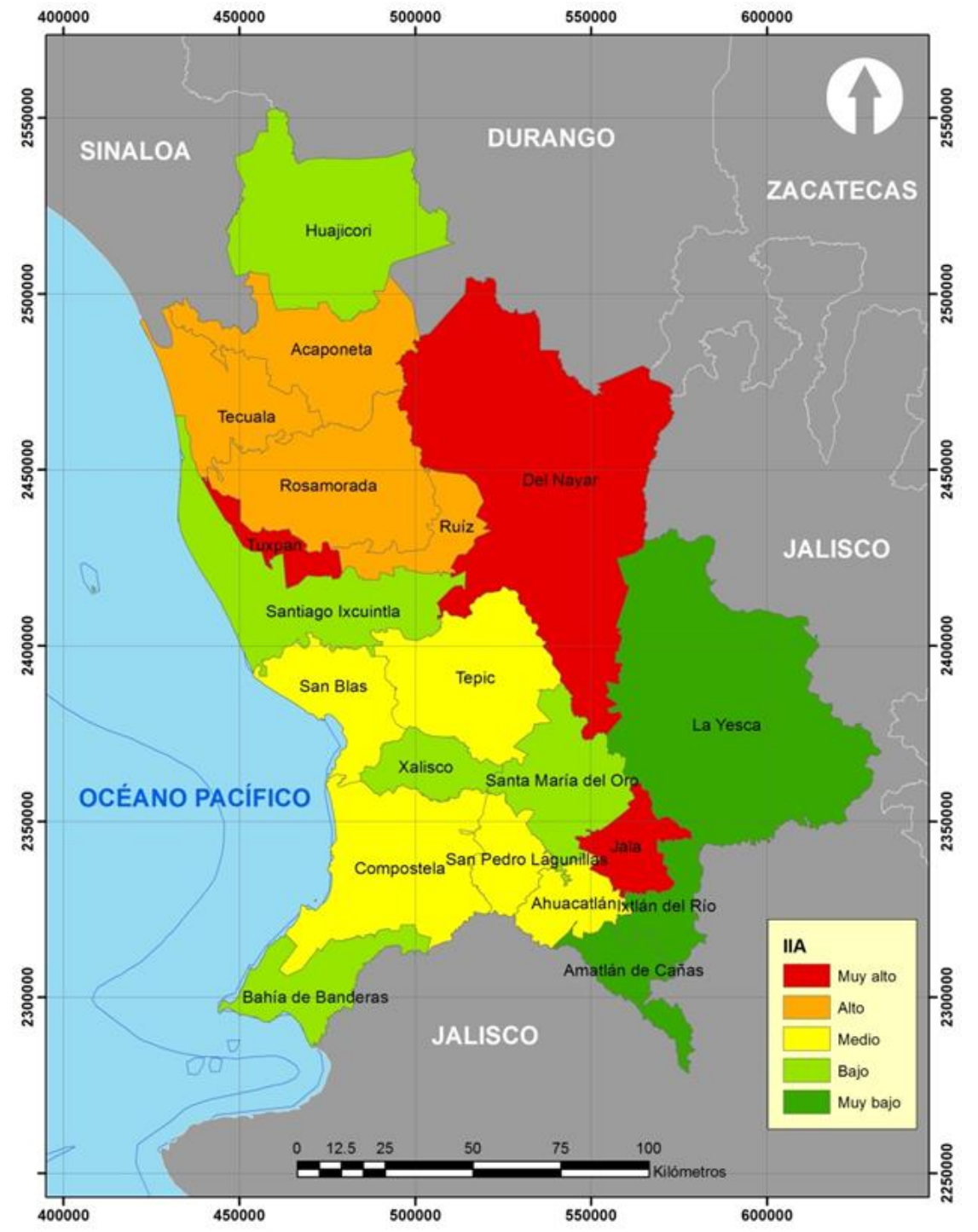

Fuente: Elaboración propia

Acaponeta presenta un ISEV alto, un IDSE bajo, un IIA alto y un IVA muy alto. La situación de desarrollo se debe a que tiene baja actividad económica: únicamente $35 \%$ de la población se encuentra económicamente activa, y además presenta alta dependencia económica (19 personas dependen de 10). En Acaponeta, $29.4 \%$ de la población reporta inseguridad alimentaria moderada o severa. Por otra parte, el IVA es muy alto debido a que mostró el índice de mortalidad infantil por desnutrición más alto del estado (8.81 caso por cada 100 000), esto lo ubica con índice de sensibilidad muy alto y no se observa capacidad adaptativa suficiente como para reducir la vulnerabilidad. Asimismo, este municipio no 


\section{Revista Iberoamericana de las Ciencias Biológicas y Agropecuarias}

presentó características de pobreza y, por ello, no se destinó suficiente apoyo por parte de programas de ayuda alimentaria.

Rosamorada presenta un ISEV alto, reporta un IDSE bajo, índices de inseguridad y vulnerabilidad alimentaria altos. La dependencia económica, un indicador del IDSE, es de 21 personas por cada 10. Asociado a ello, tiene baja actividad económica (32 \% de la población se encuentra económicamente activa). En Rosamorada, $29.4 \%$ de la población reporta inseguridad moderada o severa. Además, aunque presenta exposición y sensibilidad media, muestra baja capacidad adaptativa; registró el menor capital financiero de la entidad.

Ruiz muestra un ISEV alto, presenta un IDSE medio, un IIA alto y un IVA muy alto. El porcentaje de población con inseguridad alimentaria moderada o severa fue de 28.5. En este municipio lo que más resalta es que registró 3.99 casos de muertes por desnutrición en menores de 5 años por cada 100000 en el año 2011. Este municipio tampoco presentó características de pobreza y por ello no se destinó suficiente apoyo por parte de programas de ayuda alimentaria.

Huajicori se identificó con un ISEV alto, con un IDSE muy bajo, un IIA bajo y un IVA alto. El tema de desarrollo socioeconómico (figura 5) es lo que principalmente coloca a este municipio con un ISEV alto. Este tipo de municipios se caracterizan por tener un grado de urbanización nulo, con características rurales en la totalidad de su extensión, las cuales se asocian con un muy alto índice de marginación (1.4), con alta dependencia económica (21 personas dependen de 10) y tasa de actividad económica limitada (únicamente $32 \%$ de la población se encuentra económicamente activa), así como a que tienen un menor porcentaje (66\%) de cobertura de salud en el estado. Respecto a Huajicori, por último, se identifica la menor proporción de población ocupada con ingresos de más de dos salarios mínimos (15\%). 
Figura 5. Índice temático de desarrollo socioeconómico de Nayarit, 2015

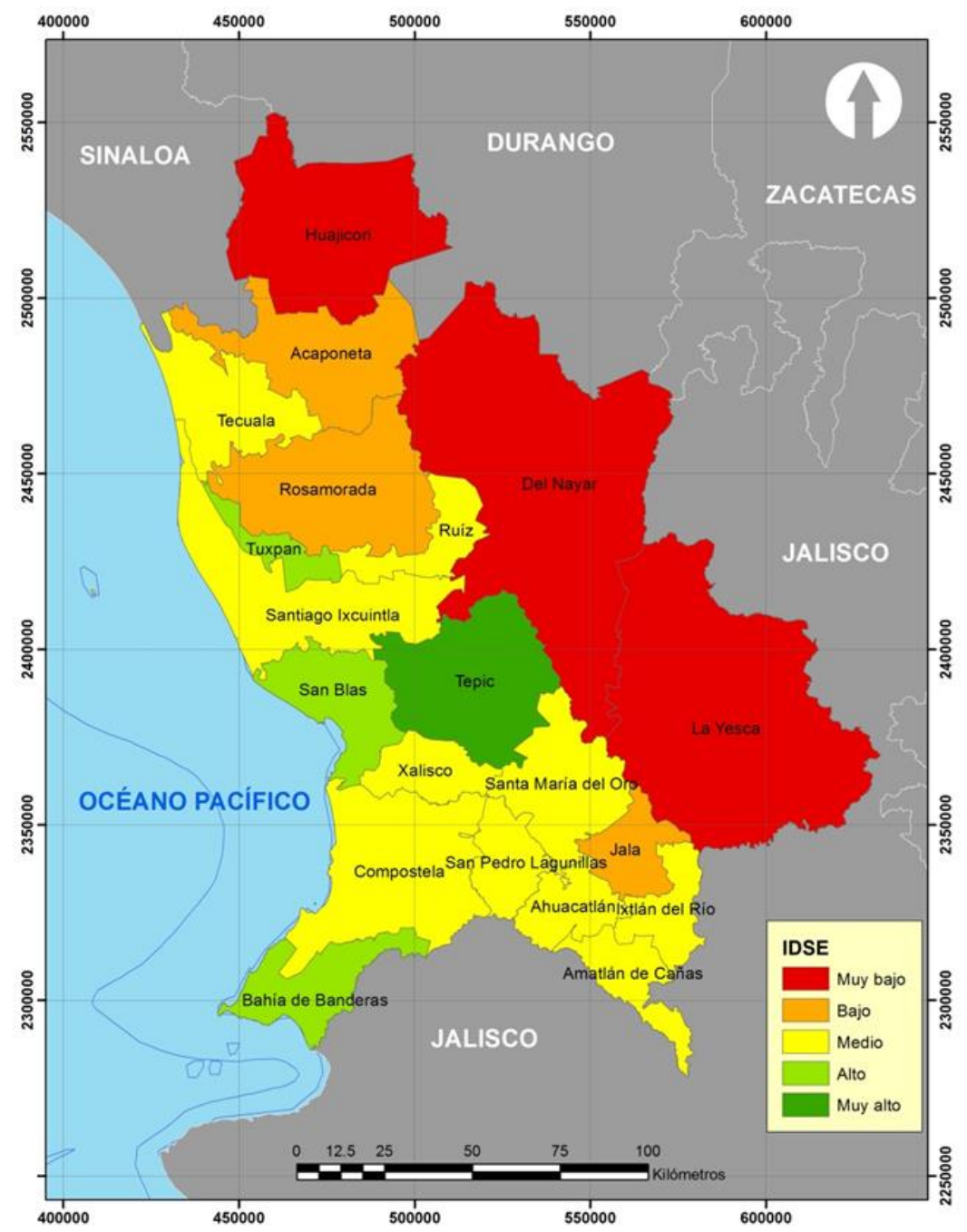

Fuente: Elaboración propia

El municipio de Tecuala presenta un ISEV medio, resultado de un IDSE medio e índices de inseguridad y vulnerabilidad alimentaria altos. Es un municipio estancado, con alto porcentaje de población con inseguridad alimentaria (27.3\%), presenta alta exposición y baja capacidad adaptativa, con alta superficie agrícola per cápita (1.25 hectáreas por persona).

En Tuxpán se observa un ISEV medio con un IDSE alto, IIA muy alto e IVA bajo. Tuxpan ocupó el tercer lugar en el estado en porcentaje de población con inseguridad alimentaria (34 \%) y población de 65 y más: aquí $10 \%$ de la población tenía 65 y más años. Resalta que solo Tuxpán se identificó con un muy alto índice de capacidad adaptativa, ya que se tuvo la mayor dotación de capital financiero; de entre los indicadores de capital financiero 


\section{Revista Iberoamericana de las Ciencias Biológicas y Agropecuarias}

destaca que tuvo la mayor tasa de apoyo económico a adultos; asimismo, del capital físico muestra la mayor proporción de tiendas de abarrotes y alimentos por hogar de la entidad.

Compostela muestra un ISEV medio, así como un IDSE, IIA e IVA medios. Este municipio es el único que mantiene todos los índices en nivel medio, no presenta características sobresalientes. Otro municipio que presenta una distribución regular de los índices temáticos que integran al ISEV es Santiago Ixcuintla con un ISEV medio, IDSE medio, IIA bajo e IVA medio.

Por sus características, La Yesca adquiere un ISEV medio. Aunque presenta desventaja con un IDSE muy bajo, se identifica un IIA muy bajo y un IVA bajo. En La Yesca se detectó bajo nivel de inseguridad alimentaria, $13.4 \%$ de la población; el promedio del estado es 25.5 $\%$. Además, este municipio se caracteriza por tener un grado de urbanización nulo (cero). Destaca porque tiene la menor densidad de población de la entidad (8 habitantes por kilómetro cuadrado). Otra característica sobresaliente es que muestra la menor esperanza de vida en el estado (60 años).

Santa María del Oro, con un ISEV bajo, presenta un IDSE medio e índices de inseguridad y vulnerabilidad alimentaria bajos. En este municipio no se presentan características relevantes.

Por su parte, Ahuacatlán muestra ISEV bajo con IDSE medio, IIA medio e IVA bajo. Este municipio presenta la mayor cobertura de agua entubada en el hogar en la entidad además no registra casos de desnutrición (año 2011).

San Blas se incluye en los municipios con ISEV bajo, presenta un IDSE alto, IIA medio e IVA bajo. En la entidad, San Blas muestra la mayor tasa bruta de actividad económica del estado (44 \% de la población es económicamente activa) y la mayor densidad de carreteras pavimentadas. La proporción de población con inseguridad alimentaria moderada y severa es de $26 \%$.

Ixtlán del Río muestra un ISEV bajo. Y los componentes de este índice se distribuyen como sigue: IDSE medio, IIA muy bajo e IVA alto. Reporta la menor inseguridad alimentaria moderada y severa en el estado (13\%). Además, este municipio presenta el índice de exposición más alto de la entidad: ocupó el segundo lugar en hogares con jefatura femenina $(28 \%)$ y tiene la esperanza de vida más alta del estado (80 años). 


\section{Revista Iberoamericana de las Ciencias Biológicas y Agropecuarias}

En Xalisco el ISEV es bajo. Presenta un IDSE medio e índices de inseguridad y de vulnerabilidad alimentaria bajos. Xalisco reporta bajo nivel de inseguridad alimentaria moderada o severa 19.5 (promedio estatal $25.5 \%$ ). Los indicadores que integran los índices temáticos no manifiestan alguna característica sobresaliente en este municipio.

Bahía de Banderas presenta un ISEV bajo, con un IDSE alto, IIA bajo e IVA medio. En este municipio se identificó la mayor concentración de las actividades económicas en los sectores secundario y terciario del estado, así como la mayor proporción (72\%) de población ocupada con ingresos de más de dos salarios mínimos. Bahía de Banderas tiene $21.4 \%$ de inseguridad alimentaria moderada o severa.

El municipio de San Pedro Lagunillas muestra un ISEV bajo, resultado de índices de desarrollo socioeconómico e inseguridad alimentaria medios, además de un IVA muy bajo. En San Pedro Lagunillas no se registraron casos de desnutrición (año 2011), presenta el menor porcentaje de población menor de 18 años (32 \%) y el mayor de población de 65 y más (12 \%) del estado, tiene las mayores coberturas de salud (87\% de la población), dotación de superficie agrícola per cápita en la entidad y producción de maíz en grano y frijol.

Tepic, la capital, muestra un ISEV bajo. Los indicadores temáticos que integran este índice tienen esta distribución: IDSE muy alto, IIA medio e IVA bajo. Este municipio registra la mejor situación de desarrollo socioeconómico de la entidad. Lo que más resalta es que muestra el índice de marginación más bajo y la densidad de población más alta en el estado; asimismo, presentó el mayor promedio de escolaridad (10 años) del estado. En el tema de capacidad adaptativa, el componente de vulnerabilidad registró el mayor capital humano de la entidad.

Amatlán de Cañas es el único municipio con un ISEV muy bajo. Presenta un IDSE medio e índices de inseguridad y vulnerabilidad alimentaria muy bajos. En este municipio lo que más resalta es que muestra el índice de exposición más bajo del estado, registra poca población menor de 18 años (33\%) y población de 65 años y más.

En este trabajo se identifica que la deficiente dotación de carreteras pavimentadas, la baja actividad económica y el bajo nivel de escolaridad del jefe o jefa de familia son factores impulsores de vulnerabilidad a la inseguridad alimentaria.

Es relevante destacar que se advierte que donde existe deficiente dotación de carreteras pavimentadas se identifica mayor vulnerabilidad a la inseguridad alimentaria; esto refuerza la 


\section{Revista Iberoamericana de las Ciencias Biológicas y Agropecuarias}

conceptualización de la seguridad alimentaria que considera, además de la disponibilidad, el acceso físico de los alimentos.

Identificar a la baja actividad económica como impulsor de vulnerabilidad es recurrente. La FAO, la Organización Panamericana de la Salud [OPS], el Programa Mundial de Alimentos [PMA] y el Fondo de las Naciones Unidas para la Infancia [Unicef] (2018) consideran que la seguridad alimentaria está relacionada con la falta de ingresos más que con la falta de alimentos, ya que, aunque exista disponibilidad de alimentos, pudiera verse restringido el acceso por no disponer de un ingreso económico suficiente en el hogar.

La vulnerabilidad de la seguridad alimentaria se refiere a toda la gama de factores que hacen que las personas queden expuestas a inseguridad alimentaria. El bajo nivel de escolaridad del jefe de familia como impulsor de la vulnerabilidad en el tema alimentario confirma que es, de origen, un problema a considerar para futuras acciones. Ya que el bajo nivel de escolaridad del jefe de familia se encuentra correlacionado con una mayor prevalencia de inseguridad alimentaria (Valencia y Ortiz, 2014; FAO, Fondo Internacional de Desarrollo Agrícola [FIDA] y PMA, 2015; Mundo, Méndez y Shamah, 2014; De Muro, 2007). De manera similar se ha reportado (Cruz y Maldonado, 2017) que el nivel de escolaridad es el principal determinante en la inseguridad alimentaria. Asimismo, Díaz, Sánchez y Díaz (2016) identifican que la escolaridad es la que más contribuye en la explicación de la inseguridad alimentaria severa en los estados de México. Se trata una capacidad que permite incrementar en las personas la oportunidad de incorporarse al sistema laboral con un ingreso suficiente para asegurar una alimentación suficiente y de calidad en los hogares. Además, el nivel de escolaridad, traducido en educación, asegura que el jefe de familia tome mejores decisiones en el consumo de alimentos de calidad nutricional.

Finalmente, el ISEV representa una evaluación actual e integral; permite identificar quiénes son más vulnerables; muestra que los municipios con alta y muy alta vulnerabilidad alimentaria global se ubican principalmente en las regiones Sierra y Norte de la entidad, en cambio los municipios identificados con muy baja o baja vulnerabilidad alimentaria global se distribuyen mayormente en las regiones Centro y Sur. Por su parte, la distribución de vulnerabilidad global media es irregular, varía según el nivel socioeconómico y educativo. Estos resultados coinciden con Seguinot (2016) en su análisis de vulnerabilidad aplicado al cambio climático; además, el nivel educativo también ha sido asociado con la inseguridad 
alimentaria (De Haro et al. 2014; De Haro y Marceleño, 2018; Valencia y Ortiz, 2014; Mundo et al., 2014).

\section{Conclusiones}

El ISEV se puede usar como instrumento para formular direcciones básicas de políticas y acciones en el tema alimentario, ya que provee información sobre dónde se localizan los más vulnerables, que son los que requieren atención inmediata. Complementariamente ofrece información sobre las condiciones que colocan a esta población en situación de vulnerabilidad y que representan un reto a vencer para los gobiernos locales. La vulnerabilidad depende de cada contexto y requiere políticas adecuadas para distintos territorios.

Hacer una observación en el tema de vulnerabilidad alimentaria a nivel municipal ha sido un gran logro debido a que existen limitantes en la disponibilidad de información, especialmente si se incluye la opinión de las personas, como se hizo en este trabajo. Pero se debe reconocer que el análisis puede ocultar una vulnerabilidad muy puntual a nivel localidad. Es posible que, en una observación más detallada, se pueda obtener información que permita focalizar y priorizar los esfuerzos institucionales a nivel localidad o menor para reducir la vulnerabilidad alimentaria en Nayarit. 


\section{Revista Iberoamericana de las Ciencias Biológicas y Agropecuarias}

\section{Referencias}

Bas, M. C. (2014). Estrategia metodológica para la construcción de indicadores compuestos en la gestión universitaria. (tesis doctoral). Universitat Politècnica de Valencia, Valencia, España.

Bengoa, J. (1996). Pobreza y vulnerabilidad. Temas Sociales, 10(4), 23-38.

Consejo Nacional de Evaluación de la Política de Desarrollo Social [Coneval]. (2017). Pobreza en los municipios de México, 2015. México: Consejo Nacional de Evaluación de la Política de Desarrollo Social. Recuperado de www.coneval.org.mx/Medicion/Documents/Pobreza_municipal/Presentacion_resultad os_pobreza_municipal_2015.pdf.

Cruz, J. y Maldonado. L. (2017). Incidencia del ingreso familiar y la educación en el acceso a la canasta básica familiar en Ecuador. Revista Económica, 3(1), 19-31.

Chaudhuri, S. and Datt, G. (2001). Assessing household vulnerability to poverty: a methodology and estimates for the Philippines. Paper presented at the $3^{\text {rd }}$ Asian Development Forum. Bangkok, June 2001.

De Haro, R. y Marceleño, S. (2018). Seguridad alimentaria en localidades rurales y urbanas en Nayarit, México. Visum Mundi, 2(1), 121-126.

De Haro, R., Marceleño, S., Garibay, G., Curiel, A. y Bojórquez, I. (2014). El alimento como eje interdisciplinario y sistémico en la educación para el desarrollo sustentable. Revista educarnos, 4(14-15), 11-26.

De Muro, P and Burchi, F. (2007). Education for rural people: a neglected key to food security. Departmental Working Papers of Economics - University Roma Tre, 0078.

Díaz, M. Á., Sánchez, M. y Díaz, A. (2016). Inseguridad alimentaria en los estados de México: un estudio de sus principales determinantes. Economía, sociedad y territorio, 16(51), 459-483.

Dumazert, P. (2008). Análisis y cartografía de la vulnerabilidad a la inseguridad alimentaria y nutricional en Nicaragua. Actualización del VAM en base a datos del periodo 2005 2008. Informe final.

Figueroa, D. (2005). Grupos vulnerables y su caracterización como criterio de discriminación de la seguridad alimentaria y nutricional en Brasil. Revista Brasileira de Saúde Materno Infantil, 5(3), 367-375. 


\section{Revista Iberoamericana de las Ciencias Biológicas y Agropecuarias}

Filgueira, C. y Peri, A. (2004). América Latina: los rostros de la pobreza y sus causas determinantes. Santiago, Chile: Comisión Económica para América Latina y el Caribe.

Freudenberg, M. (2003). Composite indicators of country performance: A critical assessment. OECD Science, Technology and Industry Working Papers, (2003/16).

Granger, W. J. (2001). Macroeconometrics - Past and future. Journal of Econometrics, 100(1), 17-19.

Gutiérrez, I. y Siles, J. (2008). Diagnóstico de medios de vida y capitales de la comunidad de humedales de Medio Queso. Los Chiles, Costa Rica: Oficina Regional para Mesoamérica y la Iniciativa CARIBE.

Holzmann, R. y Jørgensen, S. (2000). Social Risk Management: A New Conceptual Framework for Social Protection, and Beyond. Social Protection Discussion Paper, (0006).

Hughes, S., Yau, A., Max, L., Petrovic, N., Davenport, F., Marshall, M. y Cinner, J. E. (2012). A framework to assess national level vulnerability from the perspective of food security: The case of coral reef fisheries. Environmental Science and Policy, 23, 95108.

Instituto nacional de estadística y censos (2004). Análisis de la Pobreza y la Seguridad Alimentaria Nutricional en Nicaragua., Gobierno de la República de Nicaragua. INEC.

Kaztman, R. (2000). Documentos de Trabajo del IPES No 2. Aportes conceptuales. Notas sobre la medición de la vulnerabilidad social. Universidad Católica del Uruguay.

Kaztman, R. y Filgueira, C. (1999). Marco conceptual sobre activos, vulnerabilidad y estructura de oportunidades. Apoyo a la implementación del Programa de Acción de la Cumbre Mundial sobre Desarrollo Social. Montevideo. CEPAL, 7-23.

Ministerio de Desarrollo e Inclusión Social y Organización de las Naciones Unidas para la Alimentación y la Agricultura [FAO]. (2012). Mapa de Vulnerabilidad a la Inseguridad Alimentaria 2012. Ministerio de Desarrollo e Inclusión Social, Perú. Lima, Perú.

Moser, C. (1998). The asset vulnerability framework: Reassessing urban poverty reduction strategies. World Development, 26(1), 1-19.

Mundo, V., Méndez, I. y Shamah, T. (2014). Caracterización de los hogares mexicanos en inseguridad alimentaria. Salud Pública de México, 56(supl. 1), 12-20. 


\section{Revista Iberoamericana de las Ciencias Biológicas y Agropecuarias}

Organización para la Cooperación y el Desarrollo Económicos [OECD]. (2008). Handbook on Constructing Composite Indicators: Methodology and User Guide. OECD Publishing. Organización de las Naciones Unidas para la Alimentación y la Agricultura [FAO], Fondo Internacional de Desarrollo Agrícola [FIDA] y Programa Mundial de Alimentos [PMA]. (2015). El estado de la inseguridad alimentaria en el mundo. Cumplimiento de los objetivos internacionales para 2015 en relación con el hambre: balance de los desiguales progresos. Roma, Italia: Organización de las Naciones Unidas para la Alimentación y la Agricultura, Fondo Internacional de Desarrollo Agrícola y Programa Mundial de Alimentos.

Organización de las Naciones Unidas para la Alimentación y la Agricultura [FAO], Organización Panamericana de la Salud [OPS], Programa Mundial de Alimentos [PMA] y Fondo de las Naciones Unidas para la Infancia [Unicef]. (2018). Panorama de la seguridad alimentaria y nutricional en América Latina y el Caribe 2018. Santiago, Chile: Organización de las Naciones Unidas para la Alimentación y la Agricultura, Organización Panamericana de la Salud, Programa Mundial de Alimentos y Fondo de las Naciones Unidas para la Infancia.

Ozughalu, M. and Ogwumike, O. (2013). Vulnerability to Food Poverty in Nigeria. African Development Review, 25(3), 243-255.

Salomone, A. (2016). Vulnerabilidad a la inseguridad alimentaria en la ciudad de Neuquén: análisis de las políticas públicas alimentarias y las estrategias de los hogares entre 1990 y 2010. (tesis doctoral). Universidad Nacional de Córdoba, Córdoba, España.

Saltelli, A. (2007). Composite Indicators between analysis and advocacy. Social Indicators Research, 81(1), 65-77.

Seguinot, B. J. (2016). Cambio climático y vulnerabilidad de las comunidades al ascenso del nivel del mar (ANM) en la ciudad de San Juan, Puerto Rico (2005-2015). Boletín de la Real Sociedad Geográfica, tomo CLI, 239-257

Stephen, L. and Downig T. E. (2001). Getting the Scale Right: A Comparison of Analytic Methods for Vulnerability Assessment and Household-Level Targeting. Disasters, 25(2), 113-135. 
Thomson, A. (1999). Implicaciones de las políticas económicas en la seguridad alimentaria: Manual de capacitación. Roma, Italia: Organización de las Naciones Unidas para la Alimentación y la Agricultura.

Valencia R. G. y Ortiz, L. (2014). Disponibilidad de alimentos en los hogares mexicanos de acuerdo con el grado de inseguridad alimentaria. Salud Pública de México, 56(2), 154164. 


\begin{tabular}{|c|c|}
\hline Rol de Contribución & Autor (es) \\
\hline Conceptualización & $\begin{array}{l}\text { Participación igual de: Susana Marceleño Flores, Rebeca de } \\
\text { Haro Mota y Oyolsi Nájera González. }\end{array}$ \\
\hline Metodología & Principal: Susana Marceleño Flores \\
\hline Software & No aplica \\
\hline Validación & $\begin{array}{l}\text { Participación igual de: Susana Marceleño Flores, Rebeca de } \\
\text { Haro Mota y Oyolsi Nájera González }\end{array}$ \\
\hline Análisis Formal & Principal: Rebeca de Haro Mota \\
\hline Investigación & $\begin{array}{l}\text { Participación igual de: Susana Marceleño Flores, Rebeca de } \\
\text { Haro Mota y Oyolsi Nájera González }\end{array}$ \\
\hline Recursos & Principal: Susana Marceleño Flores \\
\hline Curación de datos & Principal: Rebeca de Haro Mota \\
\hline $\begin{array}{l}\text { Escritura - Preparación del } \\
\text { borrador original }\end{array}$ & $\begin{array}{l}\text { Participación igual de: Susana Marceleño Flores, Rebeca de } \\
\text { Haro Mota y Oyolsi Nájera González }\end{array}$ \\
\hline $\begin{array}{l}\text { Escritura } \quad \text { Revisión } \\
\text { edición }\end{array}$ & $\begin{array}{l}\text { Participación igual de: Susana Marceleño Flores, Rebeca de } \\
\text { Haro Mota y Oyolsi Nájera González }\end{array}$ \\
\hline Visualización & $\begin{array}{l}\text { Participación igual de: Susana Marceleño Flores, Rebeca de } \\
\text { Haro Mota y Oyolsi Nájera González }\end{array}$ \\
\hline Supervisión & Susana Marceleño Flores \\
\hline $\begin{array}{l}\text { Administración } \\
\text { Proyectos }\end{array}$ & Susana Marceleño Flores \\
\hline Adquisición de fondos & Universidad Autónoma de Nayarit \\
\hline
\end{tabular}

